\title{
Latest research progress in the correlation between baicalein and breast cancer invasion and metastasis (Review)
}

\author{
WAN-JUN YAN ${ }^{*}$, XING-CONG MA, XIAO-YAN GAO, XING-HUAN XUE* and SHU-QUN ZHANG \\ Department of Oncology, The Second Affiliated Hospital, College of Medicine \\ of Xi'an Jiaotong University, Xi'an, Shaanxi 710061, P.R. China
}

Received July 23, 2015; Accepted January 13, 2016

DOI: $10.3892 / \mathrm{mco} .2016 .750$

\begin{abstract}
Breast cancer is one of the most commonly occurring female malignant tumors. According to the 2012 GLOBOCAN statistics, produced by the International Agency for Research On Cancer ('IARC'), nearly 1.7 million women were diagnosed with breast cancer, with 522,000 related deaths: An increase in the incidence of breast cancer and associated mortality by nearly $18 \%$ from 2008 . Metastasis is the final step in breast cancer progression, and represents the most common cause of mortality in patients with breast cancer. Therefore, a search for low-toxicity, safe and effective anti-breast cancer drugs in the form of natural compounds has become an intense focus of research. Baicalein, a widely used Chinese herbal medicine, has extensive antitumor activity. The present review briefly describes the research that has been performed on the association between baicalein and breast cancer metastasis, and further illustrates the influence of baicalein on the underlying mechanisms of breast cancer metastasis, adding a novel theory basis for baicalein antitumor research. In conclusion, baicalein may represent a promising target for the prevention and therapy of breast cancer.
\end{abstract}

\section{Contents}

1. Introduction

2. Chemical structure and physiological function of baicalein

3. The function and mechanisms of anti-breast cancer induced by baicalein

4. Conclusions and perspectives

Correspondence to: Professor Shu-Qun Zhang or Miss Wan-Jun Yan, Department of Oncology, The Second Affiliated Hospital, College of Medicine of Xi'an Jiaotong University, 157 Xiwu Road, Xi'an, Shaanxi 710061, P.R. China

E-mail: zhangshuqun1971@yahoo.com.cn

E-mail: 951575470@qq.com

${ }^{*}$ Contributed equally

Key words: baicalein, breast cancer, invasion, metastasis, SATB1, EMT

\section{Introduction}

Breast cancer, which is one of the most malignant cancers in women (1), has a poor prognosis due to its high metastasis. Metastasis is the final step in breast cancer progression, and the most common cause of mortality in patients with breast cancer (2). A lack of effective treatments against breast cancer invasion and metastasis has become the major obstacle to the survival rate and quality of life in patients with breast cancer. Therefore, identifying novel drugs/compounds with an anti-invasive potential would help to control the metastatic properties of cancer cells, and consequently, searching for novel anticancer flavonoids with high bioactivities has become an intense focus for research. Notably, baicalein, a widely used Chinese herbal medicine, has historically been used in anti-inflammatory and anti-cancer therapies. However, the anti-metastatic effect and molecular mechanism(s) of baicalein in breast cancer have yet to be fully elucidated. Therefore, the present review aimed to assess the anti-metastatic effects of baicalein, and its associated mechanism(s) of action in breast cancer.

\section{Chemical structure and physiological function of baicalein}

Baicalein (5,6,7-trihydroxyflavone; BAE, molecular formula $\mathrm{C}_{15} \mathrm{H}_{10} \mathrm{O}_{5}$ ), is a flavonoid extracted from the root of Scutellaria baicalensis Georgi, a herb traditionally used in Oriental medicine. The flavonoid has been purified, with a defined chemical structure (Fig. 1), and in addition to the various pharmacological effects that it exerts, baicalein has been widely used to treat various inflammatory diseases, allergies, thrombosis and ischemia, as well as being a scavenger of oxygen free radicals (3-10). Previously, it has been shown that bacailein not only has a therapeutic effect on non-tumorous diseases, such as acute liver failure and acute myocardial ischemia $(11,12)$, but it also exerts an inhibitory effect on tumor diseases, including colorectal, prostate, liver, lung, skin, stomach and ovarian cancer (13-17). It has been reported that baicalein has wide-ranging potential antitumor effects in terms of: i) Restraining cell proliferation, invasion and metastasis; ii) promoting cell apoptosis; iii) completely inhibiting the cell cycle; iv) preventing tumor angiogenesis; v) inducing tumor cell differentiation; and vi) removing reactive oxygen free radicals and preventing the tumorigenesis $(7,18,19)$. 


\section{The function and mechanisms of anti-breast cancer induced by baicalein}

3.1 Inhibition of breast cancer cell proliferation induced by baicalein. As a predominant characteristic of cancer cells, unlimited proliferation is an important primary process in the development of tumors. To investigate the effect of baicalein on cell proliferation, MCF-7 and SK-BR-3 cells were treated with various concentrations of baicalein $(5$, 10 or $15 \mu \mathrm{mol} / \mathrm{l}$ ) for $24 \mathrm{~h}$, and cell viability was assessed using the methyl thiazolyl tetrazolium (MTT) assay (18). The $\mathrm{IC}_{50}$ values (i.e., the concentrations required to give a half-maximal inhibition) of baicalein in the MCF-7 and SK-BR-3 cells after $24 \mathrm{~h}$ were determined to be $66.3 \pm 5.9$ and $103.5 \pm 9.4 \mu \mathrm{mol} / 1$, respectively. Wang et al (20) also examined the influence of different concentrations of baicalein on the proliferation of MDA-MB-231 cells, and the rate of proliferation was revealed to be inhibited by $\sim 54.9$ and $74.3 \%$ on addition of 10 and $50 \mu \mathrm{mol} / 1$ baicalein, respectively (20). Consistently with the results of a study by $\mathrm{Xu}$ et al (21), the proliferative activity of the MDA-MB-231 cells was decreased in a concentration- and time-dependent manner. In addition, in order to investigate the effect of baicalein on cell proliferation, MCF-7 cells were treated with various concentrations of baicalein and its sulfated derivatives, and cellular proliferation was assessed using MTT, lactate dehydrogenase and flow cytometric assays, and by morphological analysis (22).

3.2 Cell cycle arrest and apoptosis of breast cancer cells induced by baicalein. Apoptosis has been defined as a discrete sequence of morphological changes, resulting in cell death with extensive double-stranded DNA cleavage, accompanied by chromatin compaction and segregation along the nuclear membrane. To investigate the effect of baicalein on cell apoptosis, MCF-7 and MDA-MB-231 cells were treated with various concentrations of baicalein, and apoptosis was assessed using a flow cytometric assay. Baicalein was demonstrated to induce cell apoptosis in a dose-dependent manner, and baicalein was shown to have the strongest proapoptotic activity in human cancer MCF-7 cells (21). Consistently with the results of a study by Zhou et al (23), the results from the flow cytometric analysis indicated that baicalein affects the survival rate of human breast cancer cells by completely inhibiting cell cycle progression in the growth (G0)/G1 and the synthesis (S)-phases. Additionally, using transmission electron microscopy, the morphological structure of the MCF-7 and MDA-MB-231 cells was observed to change when viewed under an electron microscope. The results of these experiments indicated that baicalein-induced morphological changes in the human breast cancer MCF-7 and MDA-MB-231 cells were observable as visible morphological changes associated with the processes of cell apoptosis, including cell foaming, nuclear condensation, the chromatin edge set, a certain extent of nuclear fragmentation and the formation of apoptotic bodies. The apoptotic ratio of the MDA-MB-231 cells was increased in a concentration-dependent manner. Simultaneously, the cells that were undergoing necrosis were more evident in the MDA-MB-231 cell group. The study by Lee et al (24) demonstrated a clear influence of baicalein on apoptosis-associated genes and proteins in human MDA-MB-231 breast cancer

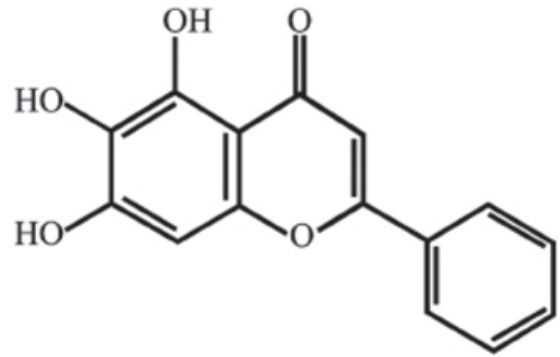

Figure 1. Chemical structure of Baicalein (molecular formula: $\mathrm{C}_{15} \mathrm{H}_{10} \mathrm{O}_{5}$; molecular weight: $270.24 \mathrm{kDa}$ )

cells (24). The results from their RT-PCR experiments demonstrated that the mRNA levels of growth arrest and DNA damage-inducible protein 153 (GADD153) increased following treatment of the MDA-M-231 cells with baicalein for $24 \mathrm{~h}$. Furthermore, western blotting indicated that baicalein increased the expression levels of GADD153, $78 \mathrm{kDa}$ glucose-regulated protein (GRP78), Bax, cytochrome $c$, caspase-3, and reduced the expression level of Bcl-2, which may have led to apoptosis in these cells (24).

3.3 Breast cancer cell migration and inhibition of invasion induced by baicalein. Breast cancer cell migration and invasion are important processes in tumor progression, and also provide indicators of tumor distant metastasis. Therefore, inhibiting tumor cell migration and invasion is also an important part in the treatment of tumor metastasis. A study by Shang et al (18) revealed that the results from the wound-healing assay indicated that the migration rate decreased to $17 \pm 2 \%$ in the MCF-7 cells, and $13 \pm 4 \%$ in the SK-BR-3 cells, following treatment with $15 \mu \mathrm{mol} / 1$ baicalein for $24 \mathrm{~h}$; the Matrigel-coated Transwell chamber system was used to investigate the effects of baicalein on the invasion of MCF-7 and SK-BR-3 cells. Baicalein was evidently able to suppress cellular invasion in a concentration- and time-dependent manner. Wang etal (20) reported that baicalein inhibited the invasive ability of the MDA-MB-231 cells in a concentration-dependent manner, using Matrigel-coated chambers. Quantification analysis indicated that invasion was reduced by $33.8,72.6$ and $87.4 \%$ when cells were treated with 2,10 or $50 \mu \mathrm{mol} / 1$ baicalein (20), respectively, consistently with the results of Gao et al (25).

3.4 Inhibition of breast cancer cell metastasis induced by baicalein. Metastasis of breast cancer cells is the major cause of morbidity and mortality worldwide. Thus, interruption of metastasis is one approach for anti-metastatic therapy (26): It is of importance for improving the survival rate and the quality of life in patients with advanced breast cancer.

Matrix metalloproteinases (MMPs). MMPs, a family of zinc-dependent proteases with well-characterized structural and catalytic properties, are able to markedly promote tumor invasion and metastasis (27). The initial step of breast cancer cell metastasis is characterized by the breakdown of the basement membrane, a process known to be dependent on type IV collagen-degrading enzymes, predominantly MMP-2 and MMP-9 (28). The expression of MMPs, particularly the 
gelatinases (MMP-2 and MMP-9), has been associated with a high potential of metastasis in several human carcinomas, including breast cancer $(29,30)$. Wang et al (20) reported that baicalein exerted inhibitive effects on the expression of MMP-2 and MMP-9 in a concentration-dependent manner. Quantification analysis indicated that MMP-9 activity was reduced by $41.6,52.7$ and $76.0 \%$, and MMP-2 activity was reduced by $12.7,41.0$ and $50.4 \%$, when cells were treated with 2,10 or $50 \mu \mathrm{mol} / 1$ baicalein (20). To further explore the exact mechanism of inhibition of invasion induced by baicalein, research conducted in our laboratory determined the expression of phosphorylated (p)-Akt, p-extracellular signal-regulated kinase (ERK), p-c-Jun terminal kinase (JNK) and p-P38 in MDA-MB-231 cells. The decrease in phosphorylation of Akt, ERK, JNK and P38 in cells treated with baicalein suggested that baicalein could inhibit the expression of MMP-2/9 through downregulation of the mitogen-activated protein kinase (MAPK) signaling pathway (20). Consistently with the results reported by Gao et al (25), immunofluorescence staining results revealed that the morphology of MDA-MB-231 cells is changed from stretching to pyknosis, and the expression of MMP-9 protein is markedly suppressed in the cell cytoplasm (21). The in vivo results reported by Ibrahim et al (31) revealed that baicalein decreased the size of the tumor volume and reduced the metastasis of the mammary gland adenocarcinoma to the lung. Additionally, their immunohistochemical staining results revealed that baicalein markedly decreased the expression of MMP-9 (31).

epithelial-mesenchymal transition (EMT). In a number of reports, EMT was observed during the initial steps of tumorigenesis and the later stage of metastasis; indeed, a strong correlation existed between EMT processes and metastatic features in cancer cells $(32,33)$. Transforming growth factor- $\beta$ (TGF- $\beta$ ) is a well-known inducer of EMT in various cancer cell types (34). In breast cancer cells, TGF- $\beta 1$ stimulates Rho-p38 MAPK-mediated EMT (36). In this process, TGF- $\beta 1$ activates Snail, Slug, Twist, zinc finger E-box-binding homeobox 1 (ZEB1) and ZEB2, which repress E-cadherin and induce EMT $(35,36)$. Chung et al $(37)$ identified that TGF- $\beta 1$ treatment induced the expected morphological changes of MCF10A cells towards the fibroblastic phenotype; therefore, their results suggested that baicalein may inhibit TGF- $\beta 1$-mediated EMT in breast epithelial cells (37). Nuclear factor $-\kappa \mathrm{B}(\mathrm{NF}-\kappa \mathrm{B})$ signaling is also reportedly required to stimulate EMT processes in various cancer types. Chung et al (37) reported that $\mathrm{NF}-\kappa \mathrm{B}$ reduces the expression level of Slug and rescues E-cadherin expression in MCF10A and MDA-MB-231 cells, and that baicalein inhibits EMT by reducing NF- $\kappa$ B. Baicalein appears to inhibit TGF- $\beta 1$-mediated EMT by reducing the expression level of the EMT-associated transcription factor, Slug, via the NF- $\kappa \mathrm{B}$ pathway. Consistently with the above-described results, baicalein also appeared to have inhibitory effects on oncogenic EMT, suppressing TGF- $\beta 1$-induced migration and colony formation in MDA-MB-231 breast cancer cells, suggesting that the baicalein-mediated inhibition of EMT may inhibit the tumorigenic activities of breast cancer cells (37). Thus, baicalein effectively inhibited EMT processes in breast epithelial cells, and this inhibitory activity effectively inhibited tumorigenic activity in breast cancer cells.

Special AT rich sequence binding protein 1 (SATB1). SATB1 is a genome organizer that tethers multiple genomic loci and recruits chromatin-remodeling enzymes to regulate chromatin structure and gene expression, and to promote tumor metastasis by regulating the expression of $\sim 1,000$ genes (38). A burgeoning number of studies have reported that the expression levels of SATB1 increased in most solid tumors, particularly in highly invasive breast cancer cells (39), and promoted the lung (40) and bone (41) metastasis of breast cancer. Baicalein inhibited the metastasis of human MAD-MB-231 breast cancer cells, possibly by reducing cell migration through downregulation of the protein expression of SATB1, indicating that baicalein is a potential therapeutic agent for human breast cancer (42).

$N F-\kappa B$ signaling pathway. $\mathrm{NF}-\kappa \mathrm{B}$, and the associated $\mathrm{NF}-\kappa \mathrm{B}$-regulated proteins, are connected with the occurrence of tumor angiogenesis, proliferation, apoptosis, invasion and metastasis (43). A study by Aggarwal et al (44) demonstrated that the treatment of MDA-MB-435 cells with baicalein eliminated the paclitaxel-induced $\mathrm{NF}-\kappa \mathrm{B}$ activation; maximum suppression was observed at a concentration of $25 \mu \mathrm{mol} / 1$. $\mathrm{NF}-\kappa \mathrm{B}$ activation by the majority of agents is known to require the phosphorylation and degradation of $\mathrm{I} \kappa \mathrm{B} \alpha$, an inhibitor of $\mathrm{NF}-\kappa \mathrm{B}$. As expected, baicalein completely suppressed the paclitaxel-mediated degradation of $\mathrm{I} \kappa \mathrm{B} \alpha$. The phosphorylation of $\mathrm{I} \kappa \mathrm{B} \alpha$ is mediated through activation of the I $\kappa \mathrm{B}$ kinase, IKK. Paclitaxel activated IKK in a time-dependent manner, and baicalein suppressed this activation. Aggarwal et al (44) also demonstrated that baicalein represses paclitaxel-induced $\mathrm{NF}-\kappa \mathrm{B}$-dependent antiapoptotic gene products [inhibitor of apoptosis (IAP)1/2, X-linked inhibitor of apoptosis protein (XIAP), Bcl-2 and Bcl-xL (37)] and paclitaxel-induced $\mathrm{NF}-\kappa \mathrm{B}-\mathrm{dependent}$ gene products [cyclin D1, c-Myc, cyclo-oxygenase (COX-2), MMP-9 and intercellular adhesion molecule-1] involved in the proliferation and metastasis of tumor cells (44). Consistently with a previous report (45), this study also demonstrated that baicalein inhibits the activation of COX-2 mRNA and promoter activity. Aggarwal et al (44) subsequently investigated the ability of baicalein to modulate human breast cancer metastasis to the lung in a nude mouse xenograft model. Their results revealed that the dietary administration of baicalein markedly decreased the incidence of breast cancer metastasis to the lung, and suppressed the expression of NF- $\kappa$ B, COX2 and MMP-9 (44). Aggarwal et al (44) also measured the proliferation rate using $\mathrm{Ki}-67$, and apoptosis using terminal deoxynucleotidyl transferase-mediated nick end-labeling assay in the tissue samples. Their results demonstrated $24 \pm 5 \% \mathrm{Ki}-67$-positive cells in the control group, and $8 \pm 4 \% \mathrm{Ki}-67$-positive cells in the baicalein-fed group. These results clearly demonstrated that the baicalein-fed group had the lowest proliferation rate. However, the terminal deoxynucleotidyl transferase-mediated nick end-labeling assay also revealed that the rates of apoptosis were not markedly different from each other $(44,45)$. Therefore, baicalein did completely inhibit NF- $\kappa \mathrm{B}$ activation and the expression of $\mathrm{NF}-\kappa \mathrm{B}-$ regulated genes in breast cancer cells, and inhibited breast cancer metastasis to the lung in nude mice. 


\section{Conclusions and perspectives}

Breast cancer is the most commonly occurring malignant tumor in women worldwide, and most patients with advanced breast cancer have serious systemic metastasis, which is associated with a high mortality rate (46). Although chemotherapy, radiation therapy and targeted therapy are able to directly kill cancer cells, certain cancer cell types are resistant to these treatments and may further proliferate and metastasize; these treatments therefore remain ineffective due to their toxicity to normal cells (47). The flavonoids are a group of naturally-occurring, low-molecular-weight compounds that are widespread in plants. Baicalein is a widely used Chinese herbal medicine that has been used historically as an anti-inflammatory agent and for anticancer therapy (48). Baicalein alone, or in combination with other herbs, has previously been shown to exert a cytostatic effect on several cancer cell lines in vitro $(49,50)$, and also in vivo $(51,52)$. Breast cancer invasion and metastasis is a complex, multistep process influenced by numerous factors, including the expression of key proteases, their inhibitory proteins, and the signaling pathways that control them. Therefore, the interruption of one or more of these steps is one approach for anti-metastatic therapy (26). Although the antitumor function of baicalein has not been investigated in clinical trials, further study of the mechanisms that underpin baicalein antitumor activity may provide possible clinical applications for the treatment of breast cancer.

\section{Acknowledgements}

The present study was supported by grants from the National Natural Science Foundation of China (grant no. 81274136), and the Program for New Century Excellent Talents in University of China (grant no. NCET-110439).

\section{References}

1. Torre LA, Bray F, Siegel RL, Ferlay J, Lortet-Tieulent J and Jemal A: Global cancer statistics, 2012. CA Cancer J Clin 65: 87-108, 2015.

2. Kim SR and Paik S: Genomics of adjuvant therapy for breast cancer. Cancer 17: 500-504, 2011.

3. Serpa R, França EJ, Furlaneto-Maia L, Andrade CG, Diniz A and Furlaneto MC: In vitro antifungal activity of the flavonoid baicalein against Candida species. J Med Microbiol 61: 1704-1708, 2012.

4. Zhang H, Tian K, Tang J, Qi S, Chen H, Chen X and Hu Z: Analysis of baicalein, baicalin and wogonin in Scutellariae radix and its preparation by microemulsion electrokinetic chromatography with 1-butyl-3-methylimizolium tetrafluoborate ionic liquid as additive. J Chromatogr A 11: 304-307, 2006.

5. Wang HW, Lin CP, Chiu JH, Chow KC, Kuo KT, Lin CS and Wang LS: Reversal of inflammation-associated dihydrodiol dehydrogenases (AKR1C1 and AKR1C2) overexpression and drug resistance in nonsmall cell lung cancer cells by wogonin and chrysin. Int J Cancer 1: 2019-2027, 2007.

6. Chen L, Dou J, Su Z, Zhou H, Wang H, Zhou W, Guo Q and Zhou C: Synergistic activity of baicalein with ribavirin against influenza A (H1N1) virus infections in cell culture and in mice. Antiviral Res 91: 314-320, 2011.

7. Li-Weber M: New therapeutic aspects of flavones: The anticancer properties of Scutellaria and its main active constituents Wogonin, Baicalein and Baicalin. Cancer Treat Rev 35: 57-68, 2009.

8. Zhang HB, Lu P, Guo QY, Zhang ZH and Meng XY: Baicalein induces apoptosis in esophageal squamous cell carcinoma cells through modulation of the PI3K/Akt pathway. Oncol Lett 5: 722-728, 2013.
9. Liu YF, Gao F, Li XW, Jia RH, Meng XD, Zhao R, Jing YY, Wang Y and Jiang W: The anticonvulsant and neuroprotective effects of baicalin on pilocarpine-induced epileptic model in rats. Neurochem Res 37: 1670-1680, 2012.

10. Zhang K, Guo QL, You QD, Yang Y, Zhang HW, Yang L, Gu HY, Qi Q, Tan Z and Wang X: Wogonin induces the granulocytic differentiation of human NB4 promyelocytic leukemia cells and up-regulates phospholipid scramblase 1 gene expression. Cancer Sci 99: 689-695, 2008

11. Wu YL, Lian LH, Wan Y and Nan JX: Baicalein inhibits nuclear factor-B and apoptosis via c-FLIP and MAPK in d-GalN/LPS induced acute liver failure in murine models. Chem Biol Interact 188: 526-534, 2010.

12. Peng Ju, Xu Wen-Jie, HE Bi-Xia, et al: Effects of baicalein on rat myocardial ischemia and neonatal cardiomyocyte injury. Chin J Nat Med 9: 132-140, 2011

13. Lea MA, Ibeh C, Deutsch JK, Hamid I and desBordes C: Inhibition of growth and induction of alkaline phosphatase in colon cancer cells by flavonols and flavonol glycosides. Anticancer Res 30: 3629-3635, 2010.

14. Niknami M, Vignarajan S, Yao M, Hua S, Witting PK, Kita Y, Shimizu T, Sved P, Patel MI and Dong Q: Decrease in expression or activity of cytosolic phospholipase A2alpha increases cyclooxygenase-1 action: A cross-talk between key enzymes in arachidonic acid pathway in prostate cancer cells. Biochim Biophys Acta 1801: 731-737, 2010.

15. Chen K, Zhang S, Ji Y, Li J, An P, Ren H, Liang R, Yang J and $\mathrm{Li} \mathrm{Z}$ : Baicalein inhibits the invasion and metastatic capabilities of hepatocellular carcinoma cells via down-regulation of the ERK pathway. PLoS One 8: e72927, 2013.

16. Wu B, Li J, Huang D, Wang W, Chen Y, Liao Y, Tang X, Xie H and Tang F: Baicalein mediates inhibition of migration and invasiveness of skin carcinoma through Ezrin in A431 cells. BMC Cancer 11: 527, 2011.

17. Wei X, Guo W, Wu S, Wang L, Huang P, Liu J and Fang B: Oxidative stress in NSC-741909-induced apoptosis of cancer cells. J Transl Med 8: 37, 2010.

18. Shang D, Li Z, Zhu Z, Chen H, Zhao L, Wang X and Chen Y: Baicalein suppresses $17-\beta$-estradiol-induced migration, adhesion and invasion of breast cancer cells via the $G$ protein-coupled receptor 30 signaling pathway. Oncol Rep 33: 2077-2085, 2015

19. Zhang K, Guo QL, You QD, Yang Y, Zhang HW, Yang L, Gu HY, Qi Q, Tan Z and Wang X: Wogonin induces the granulocytic differention of human NB4 promyelocytic leukemia cells and up-regulates phospolipid scramblase 1 gene expression. Cancer Sci 99: 689-695, 2008

20. Wang L, Ling Y, Chen Y, Li CL, Feng F, You QD, Lu N and Guo QL: Flavonoid baicalein suppresses adhesion, migration and invasion of MDA-MB-231 human breast cancer cells. Cancer Lett 297: 42-48, 2010.

21. Xu W, Xiao L, Ma Y, et al: Baicalein mediates influence of proliferation of breast cancer MCF-7 and MDA-MB-231 cells. The Chinese Medicine 14: 26-28, 2011.

22. Wang N, Ren D, Deng S and Yang X: Differential effects of baicalein and its sulfated derivatives in inhibiting proliferation of human breast cancer MCF-7 cells. Chem Biol Interact 221: 99-108, 2014.

23. Zhou QM, Wang S, Zhang H, Lu YY, Wang XF, Motoo Y and Su SB: The combination of baicalin and baicalein enhances apoptosis via the ERK/p38 MAPK pathway in human breast cancer cells. Acta Pharmacol Sin 30: 1648-1658, 2009.

24. Lee JH, Li YC, Ip SW, Hsu SC, Chang NW, Tang NY, $\mathrm{Yu}$ CS, Chou ST, Lin SS, Lino CC, et al: The role of Ca2+ in baicalein-induced apoptosis in human breast MDA-MB-231 cancer cells through mitochondria- and caspase-3-dependent pathway. Anticancer Res 28: 1701-1711, 2008.

25. Gao MH, Lin ZH, Xu YJ et al: Baicalein mediates influence of proliferation and migration of breast cancer MDA-MB-231 cells. Lishizhen Medicine and Materia Medica Research 24: 1389-1390, 2013.

26. Nam KS and Shon YH: Suppression of metastasis of human breast cancer cells by chitosan oligosaccharides. J Microbiol Biotechnol 19: 629-633, 2009.

27. Yan C and Boyd DD: Regulation of matrix metalloproteinase gene expression. J Cell Physiol 211: 19-26, 2007.

28. Lu N, Ling Y, Gao Y, Chen Y, Mu R, Qi Q, Liu W, Zhang H, $\mathrm{Gu} \mathrm{H}$, Wang $\mathrm{S}$, et al: Endostar suppresses invasion through downregulating the expression of matrix metalloproteinase-2/9 in MDA-MB-435 human breast cancer cells. Exp Biol Med (Maywood) 233: 1013-1020, 2008. 
29. Jones JL, Shaw JA, Pringle JH and Walker RA: Primary breast myoepithelial cells exert an invasion-suppressor effect on breast cancer cells via paracrine down-regulation of MMP expression in fibroblasts and tumour cells. J Pathol 201: 562-572, 2003.

30. Aye MM, Ma C, Lin H, Bower KA, Wiggins RC and Luo J: Ethanol-induced in vitro invasion of breast cancer cells: The contribution of MMP-2 by fibroblasts. Int J Cancer 112: 738-746, 2004.

31. Ibrahim A, El-Meligy A, Fetaih H, Dessouki A, Stoica G and Barhoumi R: Effect of curcumin and Meriva on the lung metastasis of murine mammary gland adenocarcinoma. In Vivo 24: 401-408, 2010.

32. Iwatsuki M, Mimori K, Yokobori T, Ishi H, Beppu T, Nakamori S, Baba $\mathrm{H}$ and Mori M: Epithelial-mesenchymal transition in cancer development and its clinical significance. Cancer Sci 101: 293-299, 2010.

33. Thiery JP: Epithelial-mesenchymal transitions in tumour progression. Nat Rev Cancer 2: 442-454, 2002.

34. Zavadil J and Böttinger EP: TGF-beta and epithelial-to-mesenchymal transitions. Oncogene 24: 5764-5774, 2005.

35. Kamaraju AK and Roberts AB: Role of Rho/ROCK and p38 MAP kinase pathways in transforming growth factor-beta-mediated Smad-dependent growth inhibition of human breast carcinoma cells in vivo. J Biol Chem 280: 1024-1036, 2005.

36. Lee JM, Dedhar S, Kalluri R and Thompson EW: The epithelial-mesenchymal transition: New insights in signaling, development and disease. J Cell Biol 72: 973-981, 2006.

37. Chung H, Choi HS, Seo EK, Kang DH and Oh ES: Baicalin and baicalein inhibit transforming growth factor- $\beta 1$-mediated epithelial-mesenchymal transition in human breast epithelial cells. Biochem Biophys Res Commun 458: 707-713, 2015.

38. Han HJ, Russo J, Kohwi Y and Kohwi-Shigematsu T: SATB1 reprogrammes gene expression to promote breast tumour growth and metastasis. Nature 452: 187-193, 2008.

39. Prat A, Parker JS, Karginova O, Fan C, Livasy C, Herschkowitz JI, $\mathrm{He} \mathrm{X}$ and Perou CM: Phenotypic and molecular characterization of the claudin-low intrinsic subtype of breast cancer. Breast Cancer Research 12: R68, 2010.

40. Minn AJ, Gupta GP, Siegel PM, Bos PD, Shu W, Giri DD, Viale A, Olshen AB, Gerald WL and Massagué J: Genes that mediate breast cancer metastasis to lung. Nature 436: 518-524, 2005.

41. Kang Y, Siegel PM, Shu W, Drobnjak M, Kakonen SM, Cordón-Cardo C, Guise TA and Massagué J: A multigenic program mediating breast cancer metastasis to bone. Cancer Cell 3: 537-542, 2003.

42. Zhang S, Gao X, Xue, X, et al: The effect of baicalein on the expression of SATB1 in MDA-MB-231 cells. Chinese-German J Clin Oncol 13: 503-508, 2014.
43. Philip S, Bulbule A and Kundu GC: Matrix metalloproteinase-2: Mechanism and regulation of NF-kappa B-mediated activation and its role in cell motility and ECM-invasion. Glycoconj J 21: 429-241, 2004

44. Aggarwal BB, Shishodia S, Takada Y, Banerjee S, Newman RA, Bueso-Ramos CE and Price JE: Curcumin suppresses the paclitaxel-induced nuclear factor-kappaB pathway in breast cancer cells and inhibits lung metastasis of human breast cancer in nude mice. Clin Cancer Res 11: 7490-7498, 2005.

45. Mukhopadhyay A, Banerjee S, Stafford LJ, Xia C, Liu M and Aggarwal BB: Curcumin-induced suppression of cell proliferation correlates with down-regulation of cyclin D1 expression and CDK4-mediated retinoblastoma protein phosphorylation. Oncogene 21: 8852-8861, 2002.

46. Parkin DM and Fernández LM: Use of statistics to assess the global burden of breast cancer. Breast J 12 (Suppl 1): S70-S80, 2006.

47. Wang S, Liu Q, Zhang Y, Liu K, Yu P, Liu K, Luan J, Duan H, Lu Z, Wang F, et al: Suppression of growth, migration and invasion of highly-metastatic human breast cancer cells by berbamine and its molecular mechanisms of action. Mol Cancer 8: 81, 2009.

48. Hatcher H, Planalp R, Cho J, Torti FM and Torti SV: Curcumin: From ancient medicine to current clinical trials. Cell Mol Life Sci 65: 1631-1652, 2008.

49. Motoo Y and Sawabu N: Antitumor effects of saikosaponins, baicalin and baicalein on human hepatoma cell lines. Cancer Lett 86: 91-95, 1994.

50. Ikemoto S, Sugimura K, Yoshida N, Yasumoto R, Wada S, Yamamoto $\mathrm{K}$ and Kishimoto $\mathrm{T}$ : Antitumor effects of Scutellariae radix and its components baicalein, baicalin and wogonin on bladder cancer cell lines. Urology 55: 951-955, 2000.

51. Ye F, Wu J, Dunn T, Yi J, Tong XD and Zhang D: Inhibition of cyclooxygenase-2 activity in head and neck cancer cells by genistein. Cancer Lett 211: 39-46, 2004.

52. Yu J, Liu H, Lei J, Tan W, Hu X and Zou G: Antitumor activity of chloroform fraction of Scutellaria barbata and its active constituents. Phytother Res 21: 817-822, 2007. 